\title{
Les Deux Étendards : libération, masturbation, profération
}

Jean-François Louette

\section{OpenEdition}

1 Journals

Édition électronique

URL : https://journals.openedition.org/recherchestravaux/714

DOI : 10.4000/recherchestravaux.714

ISSN : 1969-6434

Éditeur

UGA Éditions/Université Grenoble Alpes

\section{Édition imprimée}

Date de publication : 15 décembre 2014

Pagination : 105-122

ISBN : 978-2-84310-291-2

ISSN : 0151-1874

\section{Référence électronique}

Jean-François Louette, «Les Deux Étendards : libération, masturbation, profération », Recherches \& Travaux [En ligne], 85 | 2014, mis en ligne le 15 juin 2016, consulté le 29 octobre 2021. URL : http:// journals.openedition.org/recherchestravaux/714; DOI : https://doi.org/10.4000/recherchestravaux. 714 
Jean-François LouetTe

Université Paris-Sorbonne

\section{Les Deux Étendards : libération, masturbation, profération}

Pour Hugues Pradier, qui m'a fait découvrir ce roman

Je viens de lire pour la deuxième fois de ma vie Les Deux Étendards. Merci petit Jésus, mes yeux ont résisté à cet exploit. Lors de la première lecture, il y a quelques années, j'avais pensé rencontrer une vraie grandeur. Cette fois-ci, je suis déçu : j'ai plus été sensible à la longueur qu’à la grandeur.

Sentiments mêlés donc. D’un côté, outre l'épreuve de la longueur (plus de mille trois cents pages...), je perçois mieux comment Les Deux Étendards se situe dans le sillage des Décombres. On s'en souvient : dans ce pamphlet infâme - quoique animé d'une verve extraordinaire -, Rebatet, en 1942, se clame partisan d'Hitler, pronazi, fasciste, et se répand en flots de haine contre les juifs, fauteurs de la guerre, qu'il voue, non sans quelques fusillades (par milliers), au châtiment collectif d'un "ghetto à l'échelle du monde moderne ${ }^{\mathrm{I}}$ ", mais aussi contre les prolétaires et les communistes, et encore contre la III ${ }^{e}$ République et la démocratie parlementaire. Je reviendrai sur cette continuité entre le pamphlet à succès de 1942 et le roman négligé de $1952^{2}$. Peut-on cependant ne pas réévaluer l'objet de son étude, par le fait même qu'on s'y consacre? Embarras dont je ne prétends pas me dépêtrer.

D'un autre côté, quoi qu'il en coûte à l'admettre, un salaud antisémite, journaliste vedette de Je suis partout, collaborateur acharné, pour qui Vichy est bien trop tiède, délateur plus d'une fois - un tel homme peut être l'auteur

I. L. Rebatet, Les Décombres, Paris, Denoël, I942, p. 566.

2. Sur laquelle l'ouvrage de R. Belot est éclairant, Lucien Rebatet. Un itinéraire fasciste, Paris, Seuil, 1994 (voir les p. 368-373). 
d'un beau livre’. Aussi je ne regrette pas de m'être exposé une fois encore au feu de ce roman : "comme une buée brûlante", disait fort bien Dominique Aury en $1956^{4}$. Formule où se composent avec bonheur trois allusions : à l'épisode d'amour platonique et mystique vécu par deux des trois personnages principaux, Régis et Anne-Marie, sur la colline de Brouilly, à la poésie des brouillards de Lyon (qui n'a pas lu Les Deux Étendards ne sait pas ce qu'est cette ville), à la sensualité exacerbée du roman.

Ainsi, j'ai relu. Ma détermination n'a pas failli. Je ne suis pas tombé dans le péché. De paresse? De désintérêt pour Dieu (la fameuse acedia)? De luxure? Autant de possibilités de ne plus lire ce roman. C'est d'elles que je vais m'occuper. Bien qu'il s'agisse d'une très grande histoire d'amour, malgré la puissance de ses personnages, malgré tant d'admirables morceaux (par exemple la lettre de rupture d'Anne-Marie à Michel, ou la dernière conversation entre Michel et Régis), ces possibilités me paraissent inscrites dans le roman lui-même : comme des empêchements qui accompagnent son déploiement même. Et qui lui interdisent sans doute d'être le chef-d'œuvre que quelques-uns voient en lui's.

Le déploiement du roman, on pourrait le lire à la fois comme le récit d'une libération inachevée; comme une histoire de masturbations; comme la conquête d'une profération - d'une verve qui double le Verbe, c'est-à-dire le trahit et veut le remplacer.

\section{Une libération inachevée}

Le titre, emprunté aux Exercices spirituels de Loyola, indique les deux grandes parties du roman. Régis et Anne-Marie, les "fiancés mystiques", attirent tout d'abord Michel du côté du christianisme. Puis Michel, désabusé de l'illusion catholique, aidé par l'ultimatum que le père Rollet, son terrible directeur de conscience, a fixé à Régis, attire Anne-Marie du côté de l'agnosticisme : «je la guérirai de son Dieu» (p. 869). C'est «l'entrée de Lucifer» (p. 872), Michel se conduit en «maître de mécréance» (p. 883), jusqu’à ce qu'Anne-Marie perde la foi, et commente avec Michel, de façon très nietzschéenne, la mort de Dieu'

3. Comme dit en substance Étiemble, contre Sartre, dans «À propos de Lucien Rebatet», La Nouvelle NRF, mars 1953, repris dans Hygiène des lettres, t. II, Littérature dégagée, Paris, Gallimard, 1955, p. 204.

4. Citée par R. Belot, Lucien Rebatet..., ouvr. cité, p. 364.

5. Voir les citations par lesquelles P. Ifri fait débuter son ouvrage, "Les Deux Étendards" de Lucien Rebatet. Dossier d'un chef-d'œuvre maudit, Paris, L'Âge d'homme, $200 \mathrm{I}$.

6. Toutes les références entre parenthèses dans le corps du texte renvoient à l'édition du roman en un volume chez Gallimard, I99I. 
Or malgré la netteté de cette structure, on se lasse. Malgré le jeu de contrepoints que ménage Rebatet entre la religion et les tentations de la chair, ou les échappées de poésie (ou de contre-poésie) urbaine, malgré les variations dans la narration (récit, dialogues, lettres, fragments de journal intime), on se lasse à lire les tirades cafardes et cagotes de Régis. On n'est guère passionné par les très longues hésitations de Michel devant la foi, par les apprentissages théologiques qu'il s'inflige, par ses attardements dans l'exégèse. Il lui faut plus de 650 pages pour crever «l'illusion catholique» (p. 675). C'est beaucoup. Et comme il entreprend minutieusement la déconversion d'Anne-Marie lâchée par Régis, on n'en a pas fini avec les matières religieuses, fussent-elles celles des hérésies. Michel trouve «les monophysites, les aphtartodocètes" plus amusants que les clowns américains (p. 836). C'est son droit. Mais le lecteur n'est pas forcé d'être de son avis. Et il peine fort, par exemple, dans la longue lettre que Michel adresse à son ami Guillaume sur la façon dont il pratique la critique des Évangiles (p. 882-890), etc.

Rebatet était fort conscient de la difficulté qu'il y a, pour un romancier, à animer des idées7. Même Antoine Blondin, très favorable au roman à sa parution, parle de "quelques tunnels apologétiques ${ }^{8}$ ». Roger Nimier est plus sévère, qui, dans son compte rendu, commence par écrire que «la passion emporte tout, même l'ennui», mais ne peut se tenir d'y revenir : «De longs passages, dans le premier volume, sont très ennuyeux ${ }^{9} \%$.

Pire encore, je ne puis tout à fait écarter une critique qu'Étiemble rejette mais sans vraiment argumenter : en 1952 on a reproché au roman d'être "cucul ${ }^{\mathrm{IO}}$ ». On a même prononcé le nom de Delly. Ce qui me semble bien excessif. Mais j'ai eu par moments l'impression de lire du Claudel (plusieurs fois évoqué dans le roman), c'est-à-dire de retrouver ce côté de Claudel qui, malgré sa puissance poétique, fait doctrine : la nécessité de la transfiguration du désir, l'élévation de l'âme par le renoncement à la chair... Allons jusqu'au bout : plus d'une page relève pour moi de la nunucherie catholique. Des exemples? Régis à Michel : "C'est l'an dernier seulement, à notre seconde nuit, [...] que nous avons prié tous deux devant une petite vierge de plâtre, en lui demandant de nous fiancer pour la vie en Jésus-Christ» (p. 242). Méditation de Michel à la gare de Perrache : "Anne-Marie et Régis sont des héros et Michel est leur frère terrestre. Puisse-t-il bientôt être leur frère devant Dieu! Cette aventure de trois âmes se déroule au-delà des barrières humaines» (p. 265). Mais voici Régis

7. Voir L. Rebatet, Lettres de prison. I945-1952, Paris, Le Dilettante, I993, p. I04.

8. A. Blondin, Ma vie entre les lignes, Paris, La Table ronde, I982, p. IO3.

9. R. Nimier, Journées de lectures [1965], Paris, Gallimard, rééd. 1980, p. 240 et 243.

IO. Art. cité, p. 207. 
à nouveau : "Je viens d'avoir un entretien incomparable avec Anne-Marie. Nous avons parlé du mal. Quelle connaissance elle a de la nature humaine!» (p. 476). Et toujours lui : «Elle vient d'avoir une trouvaille sublime pour le jour de notre séparation. Ce matin-là, nous communierons tous les deux. Pendant que l'un de nous priera encore, l'autre s'en ira, pour toujours" (p. 494). Et même Anne-Marie quand elle parle de Brouilly :

Brouilly ne peut pas nous décevoir. Un autre monde commence là-haut pour nous [...] C'est parce qu'il nous a été donné d'avoir la révélation de notre sacrifice en vivant un poème unique, que nous pourrons accomplir ce sacrifice (p. 640).

Une cohorte d'anges passe.

Je sais bien la double réponse que l'on peut me faire. D'une part, Michel est le premier à dénoncer la "nigauderie de l'algèbre pieuse» (p. 615), «cette glu à l'encens» (p. 706), les "poncifs de la bigoterie» (p. 925). Il faudrait donc faire la part de la mention ironique? Peut-être. Je doute fort que tous les propos que j'ai cités plus haut soit modalisés par l'ironie du narrateur. Mais d'évidence (et d'autre part), il y a, certes, à l'égard de Régis, de l'Église comme institution, et des prêtres, une stratégie vivement satirique. La difficulté ne se dissout pas pour autant. Même quand il fait son choix contre la foi, Michel se sent ligoté à Lyon par son amour pour Anne-Marie : il vit un «martyre mesquin» - il lui sacrifie tout (p. 696). Le roman ne cesse de convoquer encore et encore ce dont il veut s'émanciper. Aux trois quarts du livre, on est encore dans la discussion religieuse. Après plus d'un millier de pages, on en arrive enfin à la sortie du christianisme (p. I048), et à la mort de Dieu (p. I055).

D'où une question : pourquoi passer tant de temps avec ce que l'on rejette? On peut supposer que Rebatet peint les deux étendards et les deux pentes de son âme, Dieu et le Diable. Mais le lecteur n'est pas obligé de suivre les deux avec le même entrain... Lorsque Michel s'interdit d'expliquer à Vignon toute l'année passée avec Régis et Anne-Marie, il formule le sentiment du lecteur :

Pouvait-on, à un Vignon, expliquer la vérité, cette logomachie, cet emberlificotage, ces incompréhensibles amours sans lit, ces cornichonneries de puceaux qui feraient sourire jusqu'à la bibliothécaire d'un pensionnat du Sacré-Cour?" (p. 685).

Habileté d'une autocritique préventive? Mais l'objet rejeté n'a-t-il pas pris le peintre à son piège? Le représenté s'installe, prend ses aises, encombre... Au grand scandale de Régis, Michel finit par lui dire en être arrivé "au point d'estimer que le catholicisme ne mérite plus le déploiement d'une telle artillerie. [...] C'est perdre son temps que de s'acharner sur lui, c'est lui attribuer une importance dont il se regonfle aussitôt. Nous avons un meilleur emploi à faire de notre vie» (p. I080-IO8I). Argument de pignouf, rétorque aussitôt Régis. Je me sens assez pignouf, sur ce plan. Et peut-être me retrouvé-je en l'heureuse 
compagnie d'Anne-Marie, qui dit à Michel : «nous nous sommes suffisamment occupés du Christ et du christianisme, nous leur avons fait beaucoup trop d'honneur" (p. 1087). Autocritique préventive encore? En tout cas, la critique n'a pas repéré ces remarques. Elles indiquent pourtant avec netteté le sentiment de plus d'un lecteur.

Mais alors, si Rebatet a lui-même vu la difficulté, pourquoi un si long débat avec le catholicisme? Pour quel bénéfice? J'entrevois trois réponses.

La première s'appuie sur la genèse du roman, écrit pour partie en prison. Rebatet écrit à Roland Cailleux qu'une "grande claustration" se situe "à l'origine de la Théologie $\|^{\text {II }}$. Sans doute fait-il allusion non pas à l'illumination de janvier 194I, qui en une nuit lui donne le schéma d'un vaste roman, mais aux deux mois passés à Moras, dans son village natal, l'été 1942, où pour s'atteler à son roman il se plonge dans une foultitude de traités d'exégèse, mystique, apologétique, théologie ${ }^{\mathrm{r} 2}$. En août 1943 , Rebatet entame la rédaction de son livre; il la poursuit jusqu'en mars I945, où il se constitue prisonnier. Parvenu à Fresnes, dans un premier temps, Rebatet ne dispose que de 200 pages de son manuscrit antérieur, sur environ 1450 ou 1550 : donc il le réécrit de décembre 1945 à début $1947^{13}$ ! Et il s'exclame : «il se pourrait bien que le meilleur eût été fabriqué ici ${ }^{14}$ !». Puis toute l'énorme dactylographie réalisée par sa femme lui parvient à Fresnes, une fois qu'il a été condamné à mort. Si bien qu'en janvier 1947, une "correction d'ensemble» est en cours; le 6 avril I947, il dit avoir "poursuivi les corrections de [s] on ours personnel jusqu'à la page I45O environ ${ }^{15}$ ». Enfin : une fois gracié, transféré à la centrale de Clairvaux, il y reçoit en novembre 1949 les 2000 pages de son roman. Ultime campagne de révision et de refonte, jusqu'en septembre 1950. Comme le note un critique : «On peut penser que l'écrivain ne donne qu'à ce moment-là toute sa cohérence et sa portée au récit ${ }^{16}$.» - Étiemble se fait de la prison une idée bien douce : «Un bon point pour la République : sa prison n'est point si cruelle qu'on n'y puisse travailler en paix ${ }^{17}$." Non sans interruptions, en tout cas. Et avec un désir farouche de liberté. Du coup, emprisonné, Rebatet se relate l'histoire d'une libération. Comme il l'écrit dans la première de ses lettres à Roland

II. L. Rebatet, Lettres de prison, ouvr. cité, p. 69.

I2. Voir P. Ifri, «Les Deux Étendards»..., ouvr. cité, p. 34.

I3. Voir les L. Rebatet, Lettres de prison, ouvr. cité, p. 32, 79, p. I04 (au moins 500 pages ont été rédigées depuis décembre 1945).

I4. Ibid., p. II7.

I5. Ibid., p. I68 et p. 220.

I6. C. Chesnot, "Les Deux Étendards de Lucien Rebatet ou l'impossible exigence du sacré», Nouvelle école, $\mathrm{n}^{\circ}$ 46, automne I990, p. 23. (Il s'agit d'une revue, dirigée par A. de Benoist, d'extrême droite - un périodique du GRECE).

I7. Étiemble, «À propos de Lucien Rebatet», art. cité, p. 208. 
Cailleux : avec la littérature surréaliste, «en tout cas, on franchit beaucoup de barreaux ${ }^{18} \%$. L'adjectif surréaliste est de trop : toute littérature vaut libération. Surtout lorsqu'elle n'est pas lue, mais écrite. Et surtout lorsqu'elle relate elle-même une libération, vécue par Rebatet à travers le personnage qui est le plus proche de lui, Michel ${ }^{19}$. Que le catholicisme lui apparaisse comme une prison, c'est l'évidence (textuelle) même : Régis est «fermé à triple tour dans sa voiture cellulaire qu'il prenait pour le véhicule de l'idéal» (p. 672), il est "cadenassé» (p. 828), comme les catholiques de façon générale : "Quels êtres cadenassés!» (p. 865), et les pères de l'Église réclament «toujours davantage de flics, de juges et de prisons pour le service de leur dieu " (p. 889). Michel se félicite qu'Anne-Marie soit enfin délivrée de la "chaîne» des prétendues vérités de l'Église (p. 904), de la «cage chrétienne» (p. 905), du "cachot chrétien» (p. I088). Trop long débat avec le catholicisme? Oui, mais tant qu'il s'explique avec lui, Rebatet, à défaut de s'évader, scie jour après jour les barreaux d'une prison mentale et sociale.

Pourtant, tout en se déclarant "agnostique absolument impénitent », et tout en refusant les «secours religieux», Rebatet, qui pense être exécuté à l'aube, indique au prêtre qui vient le visiter dans cellule de Fresnes qu'il souhaite "la présence auprès de [lui] d'un représentant du christianisme, dans [s]es dernières minutes"; du coup, il distingue entre l'agnosticisme de l'individu, et l'opinion de «l'homme social» en lui, homme qui «estime finalement que le christianisme demeure irremplaçable, qu'il apparaît assez chimérique de prétendre sauver notre monde sans son secours ${ }^{20}$ ». Ainsi la longue libération n'irait pas jusqu'à son terme?

Une lecture critique s'est engagée dans ce sens. Christophe Chesnot, dans une revue d'extrême droite hélas, a soutenu : I) Que puisque notre modernité «réside dans l'absence du sacré», dans «l'impossibilité de donner une dimension sacrée à la vie dans le monde actuel ", du coup, plus Dieu disparaît de nos sociétés, plus le roman de Rebatet serait actuel. Et surtout : 2) Que le personnage capital, celui qui confère sa vraie grandeur au roman, n'est pas Michel, mais Anne-Marie, qui serait le "véritable porte-parole de l'écrivain $»^{21}$. C'est elle qui dessinerait la troisième voie : ni Dieu ni Diable (un des titres envisagés pour le roman), mais l'exigence maintenue de donner une dimension sacrée à la vie. Le mot de la fin, dans le roman, appartient à Régis : "Mais moi, je lui [Anne-Marie] laisserai un souvenir lumineux. " Il ne signe certes

I8. L. Rebatet, Lettres de prison, ouvr. cité, p. I9.

19. Ibid., p. 193.

20. Voir l'extraordinaire lettre écrite pour relater sa «résurrection», le jour de Pâques 1947

(L. Rebatet, Lettres de prison, ouvr. cité, p. I80 et I9I).

2I. C. Chesnot, "Les Deux Étendards...", art. cité, p. II, 30, 2I. 
pas sa victoire (on peut le trouver erroné ou mensonger, et Rebatet félicite un journaliste d'y avoir décelé «un orgueil très grinçant ${ }^{22}$ »), mais il pourrait quand même désigner une valeur : ce souvenir lumineux, dirais-je, est aussi numineux, et il rejoindrait la valeur de l'épisode de Brouilly - qui serait d'avoir fait sentir l'union de Dieu et des hommes au sein même du monde. Il faudrait alors - je complète l'article sur lequel je m'appuie - aller jusqu'au bout de cette logique. Dans Les Décombres, Rebatet indiquait son désir d'arracher la jeunesse à l'Église : «dans l'état de judaïsation et d'anarchie où on la voit, il est impossible de lui consentir cette faveur ${ }^{23}$ " (laisser l'Église catholique préparer la jeunesse pour ses desseins futurs). Aussi le pamphlétaire appelle-t-il de ses vœux une réforme de l'Église (et non pas un radical adieu au christianisme). Position qui préfigure celle de... Régis se proposant de «restaurer la chrétienté déclinante» (p. 395). Les Deux Étendards, dont Rebatet dit expressément qu'il est écrit pour les jeunes gens ${ }^{24}$, serait en ce sens, au moins pour partie, un roman didactique : pédagogique et à thèse. Mais il a l'habileté de procéder avant tout par la satire (Michel), et de ne laisser qu'entrevoir (chez Régis et chez AnneMarie) exigences de sacré ou de restauration de la chrétienté.

Or une telle lecture ne me convainc guère. Son réel mérite est de donner toute sa grandeur au personnage d'Anne-Marie. Mais sans doute celle-ci demeure-t-elle plus ambiguë (plus sensuelle, et plus désespérée pour finir) que ne le veut Chesnot. D'autre part, plus que vers un autre sacré (non catholique), le roman se tend sans doute, comme l'a suggéré Yves Reboul, vers une «révolution nietzschéenne des valeurs ${ }^{25} »$, plus dionysiaque que compatible avec un christianisme même remanié. Enfin, une telle interprétation ne répond guère à l'objection pignouf que j'ai présentée (trop de religion dans tout ça!). Car pourquoi serait-on forcé de continuer à contempler la mort de Dieu, tout en rêvant d'une re-sacralisation du monde? N'est-ce pas, en un sens, une affaire réglée en I95I - avec la mort de Gide, et avec la parution du Diable et le Bon Dieu (qui a le bon goût d'être - quoique long pour une pièce de théâtre - plus court que Les Deux Étendards). Rebatet tirerait sur une cible qui serait déjà morte, ou du moins mourante, et de plus en plus désuète? Il lui arrive de s'étonner devant un homme totalement "a-chrétien ${ }^{26} »$. Mais si tel était l'état qui s'installe progressivement en France après la Seconde Guerre mondiale, et qui prévaut, désormais? Pas de nostalgie du sacré à avoir. (On a pu faire le même reproche à Bataille). Ce

22. L. Rebatet, Lettres de prison, ouvr. cité, p. 270.

23. L. Rebatet, Les Décombres, ouvr. cité, p. 560.

24. Dans une lettre à un lecteur citée par P. Ifri, ouvr. cité, p. 5 I.

25. C'est la lecture de Y. Reboul dans «Lucien Rebatet : le roman inachevé», Études littéraires, vol. 36, n ${ }^{\circ}$ I, été 2004, Ecrivains encombrants, p. 13-29.

26. L. Rebatet, Lettres de prison, ouvr. cité, p. I6I. 
conflit n'est plus le nôtre. On ne peut plus penser comme Étiemble : «Michel, c'est notre jeunesse ${ }^{27} »$. Ce décalage n'ôte certes pas tout intérêt au roman. Mais sous cet angle il s'est éloigné de nous, et il peut ennuyer.

Pourtant, si Rebatet a entrepris une aussi longue bataille romanesque contre le catholicisme institutionnel, c'est enfin (troisième réponse possible) parce qu'il veut poursuivre la guerre qu'il menait dans le chapitre consacré par Les Décombres à "La religion chrétienne». Le roman, décidément, s'inscrit dans le droit fil du pamphlet. Non seulement parce qu'après les décombres de la France, Rebatet peint, à travers l'échec amoureux de ses trois personnages, "les ruines de tout bonheur humain» (telle est la chute de la quatrième de couverture); mais surtout parce que le pamphlet fournit une part essentielle de l'intertexte doctrinal du roman ${ }^{28}$. Chez le Michel de 1952 et chez Rebatet en 1942, même rejet du troupeau et même éloge de Nietzsche. Surtout, Michel raille et le "rabbi Jésus» (p. 902), et saint Paul, "ce juif génial et terrible» (p. 887), tout comme le pamphlétaire prononçait une âpre condamnation $\mathrm{du}$ «judéo-christianisme», dont l'admiration de l'Église pour Bergson serait un symptôme (et dans le roman le Père Chaleyssin, qui doit faire franchir à Michel le pas ultime vers la foi, est défini comme un "éminent bergsonien", p. 633). Les Deux Étendards ne viseraient-ils si obstinément le catholicisme que parce que Les Décombres ont prétendu montrer à quel point il s'est "enjuivé»?

\section{Le roman d'Onan}

Tout cela est assez emberlificoté. Et les jeunes gens d'aujourd'hui sont peutêtre plus séduits par les descriptions répétées de la belle Anne-Marie que par la perspective d'une restauration de la chrétienté ou du sacré. Reste donc l'autre étendard. Soyons bordelier comme Rebatet dit savoir l'être : celui qui étend son dard.

Or, là aussi, une difficulté se présente, dont Rebatet était bien conscient : "Anne-Marie ne peut pas se faire baiser au débotté par Michel, sans que l'un et l'autre de ces partenaires ne déchoie [sic] de plusieurs crans "; d'où la nécessité, dans tout le deuxième mouvement du roman (après la rupture entre Régis et sa belle), de «broderies", de retardements ${ }^{29}$. Et le lecteur les sent comme tels (par exemple l'épisode lesbien de la Chauve-souris, malgré ses qualités).

27. Article cité, p. 2IO.

28. Il est donc fort contestable de prétendre, comme P. Ifri, que le roman n'est à considérer que "du seul point de vue littéraire» (ouvr. cité, p. 13).

29. L. Rebatet, Lettres de prison, ouvr. cité, p. 78 et 8O-8I. 
Le roman se paralyse quelque peu, dans ces conversations toujours reprises avec Anne-Marie : "Michel s'évertuait à ramener les thèmes familiers [les contradictions du catholicisme], mais sa verve demeurait factice» (p. II27). Au moins, chez Drieu la Rochelle, on couche vite - sauf dans Blèche, dont le protagoniste n'est pas un journaliste catholique et tartuffe par hasard -, et les problèmes commencent après.

Hypocrite lecteur, mon frère, reconnais-le : ce sont pourtant toutes ces pages animées par le désir sexuel qui rendent le roman mémorable - les jeunes filles vues par Michel depuis le café «La Source», la tentation qu'il surmonte chez Yvonne Ageron, la jeune Gaupette filée dans la nuit lyonnaise, les progrès (assez lents, donc) vers l'amour physique entre Michel et Anne-Marie.

Mémorables pour le lecteur... et pour l'auteur? C'est en prison qu'il rédige "les chapitres les plus chauds du second tome», selon Pol Vandromme ${ }^{30}$. Je voudrais ici introduire une hypothèse dont on ne doit pas se scandaliser trop vite : Les Deux Étendards est le roman d'Onan.

Il ne s'agit naturellement pas de soutenir que Rebatet se manuélisait en écrivant ce livre. Nous n'en savons rien, et évidemment peu nous importe. Il avoue certes que la rédaction de certaines pages le met «rapidement dans un état superphallique», et qu'un de ses compagnons de prison «s'empoigne le vit» en l'entendant lire tel épisode; il a conscience de frôler la "littérature masturbée $e^{31}$. Mais on peut lui faire crédit quand il déclare trop respecter la littérature pour en faire un "chatouille-gland»; il se défend aussi d'avoir sombré dans «l'onanisme des geôles» en écrivant les deux cents pages qui conduisent Anne-Marie et Michel dans le même lit ${ }^{32}$. Dénégation? Là encore, peu importe. En revanche, il s'agit de prétendre qu'Étiemble avait tort de tonner contre «le porc qui écrivit que Rebatet ne décrit si longuement les jeux d'amour que pour se branler, prisonnier, l'imaginaire». Parce que cette coquine (je n'ose dire coquette) opération répond exactement à la fois à la conception de l'écriture que Rebatet développe dans ses lettres de prison, et à celle que le roman lui-même présente.

En effet, de façon générale Rebatet ne peut écrire son roman que si les conditions suivantes sont réunies (et il l'écrit aussi pour les réunir) :

I) La bride ne saurait être lâchée à la verve sans documentation : «journaux intimes [de jeunesse], lettres, notes prises sur le vif ${ }^{33}$ ». C'est le substrat autobiographique du roman, l'histoire vécue en 1925-1926, que Rebatet suit

30. P. Vandromme, Rebatet, Paris, Éditions universitaires, I968, p. II7.

3I. Je remercie N. d'Estienne d'Orves pour m'avoir autorisé à citer ces fragments d'un texte inédit de Rebatet, "Étude sur la composition des Deux Étendards».

32. L. Rebatet, Lettres de prison, ouvr. cité, p. Io6 et I07.

33. Ibid., p. 33. 
de près $^{34}$ : Régis transpose l'ami François Varillon, qui deviendra en effet jésuite, Anne-Marie réinvente Simone Chevallier. Le roman est élaboration de souvenirs.

2) L'auteur entretient une extrême proximité avec ses personnages. L'amour physique avec Anne-Marie doit être écrit "dans un état de passion réelle»; écrire les deux cents pages décisives pour ce qui est des relations entre Michel et Anne-Marie a mis Rebatet "dans l'état physique et plus encore moral d'un amoureux $x^{35}$ ". Amour et désir qu'on sent à lire le roman.

3) L'écriture elle-même est une activité très fortement sexualisée. Je ne peux "absolument pas m'en passer", dit Rebatet, mais c'est un phénomène qu'on ne saurait réglementer : un soir "ça gicle dans tous les sens", le lendemain on se relit, c'est mauvais; ce qui est difficile, c'est de poursuivre : «On tire d'abord son coup, un, ou plusieurs, ou des quantités", mais cette période du "projet initial» ne donne pas toujours lieu à "fécondation"; heureusement, la chasse au mot juste demeure une "voluptés ${ }^{36}$.

Bref : l'écriture des Deux Étendards renouvelle cette «intense activité fantasmatique $^{37}$ " que Rebatet avait connue en 1925, à la suite de la rencontre avec Simone Chevallier. On le sait bien : Freud en 1905 définissait les fantasmes comme des «représentations qui ne sont pas destinées à se réaliser»; et en 1908 il a suggéré la continuité entre la création de fantasmes ou fantaisie (das Phantasieren), les rêves diurnes, et la création littéraire ${ }^{38}$. Le roman s'écrit dans une fièvre imaginaire et charnelle, par giclées de désir. L'enjeu est notamment de corriger ou de compenser le passé vécu : à l'issue de sa relation avec Simone, Rebatet est encore puceau, il ne l'a pas possédée ${ }^{39}$. Triste ratage : "on rate des femmes. On rate aussi sa vie», pense Michel (p. II25). Mais le roman permet de revivre, d'intensifier, et de modifier les souvenirs : à Paris, Michel a déjà, lui, connu plus d'une femme; Rebatet regrette de ne pas avoir eu (comme disait Stendhal) Simone : Michel non seulement l'aura (devenue Anne-Marie), et la fera jouir, mais il nous est peint comme se payant le luxe de renoncer à plusieurs conquêtes tout acquises (sa cousine Marie-Louise, Yvonne Ageron, telle rousse charnue à l'hôtel Carlton). La première cellule de Rebatet à Fresnes

34. R. Belot parle de sa "fiabilité autobiographique [...] rarement mise en défaut» (Lucien Rebatet..., ouvr. cité, p. 68).

35. L. Rebatet, Lettres de prison, ouvr. cité, p. 37 et I07.

36. Ibid., p. 87-89.

37. R. Belot, Lucien Rebatet..., ouvr. cité, p. 78, à propos de l'année 1925 et d'un «carnet couvert de dessins de filles nues".

38. Voir les Trois essais sur la théorie de la sexualité, et «Le créateur littéraire et la fantaisie» (ou «La création littéraire et le rêve éveillé», selon une autre traduction) dans L'Inquiétante Etrangeté et autres essais.

39. Voir R. Belot, Lucien Rebatet..., ouvr. cité, p. 83-84. 
porte le numéro 348. Ce sera aussi le numéro de la chambre où Michel possède enfin Anne-Marie : l'espace de la prison fonde l'espace romanesque érotique qui le recouvre et le transforme.

Que nous dit maintenant le roman, si l'on décide de s'en tenir à lui ? Notons tout d'abord qu'il ne cesse de convoquer, comme un leitmotiv assez peu wagnérien, le thème de la masturbation. Tantôt à propos de personnages secondaires : de telle couturière vue dans un train, Michel pense élégamment qu'elle «s'asticote régulièrement la cramouille» (p. 843); les pages consacrées aux séances nocturnes d'onanisme auxquelles s'adonne Mlle Denise, et que Michel entend de sa chambre, comptent parmi les morceaux de bravoure du roman. Tantôt à propos de Michel lui-même. Les moments de continence ne vont pas sans pollutions nocturnes, et c'est le thème des "cartes de géographie» repérables sur les draps (p. 409 et 558) : «singulière puissance des rêves charnels!» (p. 698). Parti pour une retraite dans sa famille, Michel ne peut oublier le carambolage frôlé d'Yvonne. Dénotation : "Je me mis à me tirer le membre impétueusement [...]» (p. 513). Connotation : "Je ne suis pas très fier d'avoir donné l'autre nuit le branle à tout un peuple de démons en l'honneur de cette petite pintade d'Yvonne» (p. 519). L'épisode de Gaupette fournira la matière d'un "hallucinant cinéma» (p. 730), formule savoureuse puisqu'on connaît le goût de Rebatet pour le septième art; Michel imagine ce qui se passe avec elle dans le «bouge à sidis" (p. 722, sic) : "Je suis un moment sur le point de me taper un rassis, dans le noir, contre le mur» (p. 726). Il lui arrive de céder aux suggestions de Mlle Denise : "Je l'ai accompagnée par trois fois» (p. 733). Anne-Marie suscite elle aussi la tentation de la masturbation : «Non, cette nuit encore, il ne céderait pas» (p. I06I).

Pourquoi une telle insistance? J'entrevois deux raisons. D'une part, il s'agit évidemment de faire pièce au versant religieux du roman, dont Rebatet sait tout le poids. Tel est le rythme de fond des Deux Etendards : religion/excitation, mystique/érotique. (Il faudrait certes ajouter l'esthétique, et notamment tout le discours assez dionysiaque sur la musique - mais, nouvel aveu, je le trouve assez ennuyeux, fait de "tartines» digressives...). Roman de la recherche de Dieu, roman de la polémique contre l'Église, certes, mais Michel note aussi que la relation de la scène - le carambolage frôlé - avec Yvonne «tiendrait une honorable place dans la littérature à ne lire que d'une main» (p. 5I2), propos qui vaut aussi bien pour tout un pan du roman. Après la peinture des plaisirs que se donne Mlle Denise, une note prête au même Michel ce commentaire : «N'ai-je pas pris moi-même plaisir à outrer ce passage?» (p. 703). Et l'auteur donc! Michel cette fois dit ne pas céder à la tentation onaniste, pour trois raisons : la masturbation est le vice chrétien; elle est insatisfaisante; enfin, 
«la masturbation est trop littéraire» (p. 705). Quel aveu - d'un lien entre littérature et onanisme!

Et c'est la seconde raison que j'annonçais : l'onanisme dans le roman, qu'il s'accomplisse ou non, importe dans la mesure où il fait intervenir exactement les mêmes composantes que l'écriture selon les Lettres de prison. À savoir, on ne l'a pas oublié : la réclusion + le souvenir + le désir et le corps + l'imagination. La masturbation est «le vice des reclus» (p. I250). Il arrive à Michel de patauger "dans la plus morose obscénité»: pris entre le souvenir d'une belle boulangère qu'il a connue charnellement, et son "imagination [qui] travaille» au sujet de n'importe quelle "péronnelle à lunettes» (p. 713). Mais c'est le roman tout entier qui, comme de juste, naît de ces deux instances, la mémoire et l'imagination. Et l'écriture elle-même est présentée par Michel comme une activité corporelle, voire comme une forme d'onanisme, selon la même sexualisation qu'on a repérée dans les Lettres de prison. Voici Michel "poussé vers sa table par le jet de sa méditation, l'achevant sous sa lampe, le poing ferme et vainqueur" (p. I70). Voici encore, dans le décisif chapitre vir, intitulé "L'Enfance de l'art», où Michel conçoit le projet de raconter l'histoire du trio ${ }^{40}$, une série d'expressions significatives : il faut "empoigner» l'œuvre (p. I80), «faire des muscles à sa phrase» (p. I8I), «rester le maître de sa main» (p. I83), savoir que «le travail, comme l'amour serait un maître féroce", payé au mieux d'un "éclair de fierté, aussi bref que l'autre spasme» (p. I90). Et voici enfin que Michel, toujours amoureux fou d'Anne-Marie, a l'idée d'écrire une lettre à son ami Guillaume pour se délivrer de son bouillonnement intérieur : mais cet « exutoire littéraire " ne serait qu'un "autre onanisme» (p. 929).

On peut donc définir la création romanesque selon Rebatet comme un exercice onaniste qui implique, je le répète, le souvenir, le désir physique, l'imagination, la main, et ce liquide qu'est... l'encre. Cet exercice ne va pas sans rappeler les pratiques d'un illustre embastillé : le marquis de Sade ${ }^{41}$. Par ailleurs, inutile de souligner que Les Deux Étendards l'oppose implicitement au texte célèbre auquel il emprunte son titre : les Exercices spirituels d'Ignace de Loyola, référence absolue pour Régis. C'est lui qui les fait découvrir à Michel; il s'en inspire lui-même - «mes gribouillis sont aussi des exercices» (p. 306), et finit par donner l'ouvrage à Michel : "un manuel miraculeux» (p. 546) sur lequel modeler sa vie entière. Or Michel rompant avec le catholicisme ne

40. Et où selon Chesnot «l'écrivain se regarde écrire» ("Les Deux Étendards...», art. cité, p. 26).

4I. Voir les belles analyses de M. Foucault sur les «frénésies solitaires», fantasmagories sexuelles, et "principe de la jouissance répétée» à l'infini qui définissent l'écriture chez Sade, "Conférences sur Sade» [1970], La Grande Étrangère, Paris, Éditions de l'École des hautes études en sciences sociales, 2013, p. I59-165. 
peut que rompre aussi avec Loyola : il parle alors d' «ignaceries» (p. 667), de "l'écumoire de saint Ignace» (p. 670), il traite le procédé de la composition de lieu d' "ignoble machinerie» (p. 967), et il en arrive à déclarer que les Exercices sont "l'école du soldat appliquée à l'esprit", dressé à un "automatisme de grenadier prussien" (p. I230). Il ne lui reste qu'à pervertir le modèle ignacien : aussi pratique-t-il la composition de lieu, mais à propos d'AnneMarie, s'efforçant de "recomposer de mémoire" sa chambre où il n'a passé que quelques instants (p. 785). De même que la méthode de Loyola implique une élaboration fantasmatique, un travail d'imagination à partir de souvenirs (pieux), de même pour Michel dans sa passion pour Anne-Marie : «sa passion se gorgeait d'inépuisables souvenirs» (p. 665); et cette passion est hantée par le désir - le Sexe étant défini comme un "assaut, continu, acharné d'images» (p. 703). Sur le point de dépuceler Anne-Marie, Michel s'apostrophe : "Cette image qui ne mourra qu'avec toi : ta fille, les yeux clos, sans un mouvement de défense, offrant son sexe ouvert» (p. II94). Lui s'offre ce qu'il lui était arrivé de s'interdire auparavant : un arrêt sur image (p. 856). On ne saurait mieux dire que, comme le souvenir, l'imagination est dans Les Deux Étendards un mode de possession charnelle.

Ainsi Michel opère-t-il un geste de libération décisif : détourner les exercices spirituels du côté de l'imagination érotique. On peut soupçonner que c'est aussi celui de Rebatet : il raconte comment, aux jours les pires de son incarcération (en Allemagne), il a «reconstitué panneau par panneau certaines salles des Offices", ou encore comment, la nuit du vendredi saint, pour s'endormir (alors qu'il craint d'être exécuté), il se livre à un de ses "passe-temps favoris de cet instant-là : composer quelques salles de musée avec mes tableaux préférés ${ }^{42}$ ». Où se rejoignent composition de lieu, composition d'art, et jouissance esthétique.

Je parlerai donc d'un régime masturbatoire de l'écriture dans Les Deux Étendards. Je le répète, il ne s'agit pas du tout, par là, de le déprécier : ce qui rend le roman fascinant c'est la charge de désir qui s'y exprime; Alain définissait le romanesque - en 1935, dans En lisant Balzac - comme «l'invraisemblable rencontre du désir et de l'événement" : en ce sens le roman est tout entier tendu, ou magnétiquement orienté, vers le moment où Anne-Marie finit par se donner à Michel. Mais l'on peut appliquer à Rebatet ce qu'il écrit, dans Les Décombres, de Victor Hugo âgé : il a su «tromper ses vieilles envies en pelotant amoureusement ses mots ${ }^{43}$ ».

On devrait aussi introduire deux points de comparaison (outre, donc, Sade). D'une part, avec la genèse des Trente-trois sonnets composés au secret par

42. L. Rebatet, Lettres de prison, ouvr. cité, p. I36, et 176.

43. L. Rebatet, Les Décombres, ouvr. cité, p. II4. 
Jean Cassou, emprisonné à Toulouse par les nazis pour faits de résistance, et qui se livre, fin I94I-début 1942, à une "composition purement cérébrale et de mémoire" (ce sont ses mots dans une préface de 1962). Guère d'érotisme pourtant dans ces beaux poèmes. Mais surtout, les modalités de la création des Deux Etendards pourraient être éclairées par un livre qui paraît lui aussi en 1952, et qui est signé d'un écrivain auquel Rebatet trouve «bougrement du talent!44 » : le Saint Genet de Sartre. Formidable essai où Sartre (est-il le porc que visait Étiemble?) s'interroge sur les liens entre emprisonnement, masturbation, imaginaire, écriture. Voici ce qu'il écrit à propos de Notre-Dame des fleurs, composé... en prison par Genet, et présenté par Sartre comme «l'épopée de la masturbation", ou comme "le recueil des talismans érotiques de Genet», c'est-à-dire des phrases qui réussissent à l'émouvoir :

Les mots qui composent ce livre sont ceux qu'un détenu s'est dit en haletant, ceux dont il s'est lesté comme de pierres pour s'alourdir et couler à pic au fond de son rêve, ceux qui naissaient du rêve lui-même et qui sont des mots rêvés, des rêves de mots ${ }^{45}$.

Il ne s'agit pas (non plus) pour Sartre de ramener tout le livre de Genet à des scénarios masturbatoires; certaines séries d'images chez Genet (ou Rebatet?) ont bien dû fonctionner ainsi, d'autres ne sont que des "possibles romanesques" : "toutes les images qu'il a caressées sans jouir", mais qui lui ont procuré un assouvissement verbal ${ }^{46}$.

Tentons à ce point tenter d'articuler les deux versants du roman de Rebatet. Le pire que Michel, tout compte fait, reproche à la religion catholique, dans son ultime conversation avec Régis, tient peut-être à ceci : «Les hommes n'étaient pas assez solitaires. Il a encore fallu que votre Dieu s'en mêlât pour les rendre définitivement incompréhensibles les uns aux autres» (p. 1308). La religion n'a rien relié. Elle a séparé. Régis ne convainc pas Michel, Michel ne convainc pas Régis. Brouilly aboutit à une brouille. Ni Régis ni Michel ne gardent Anne-Marie. Chacun est dès lors renvoyé du côté de ses exercices spirituels, orthodoxes pour Régis, hétérodoxes pour Michel ou pour Rebatet : l'écriture onaniste.

44. Ainsi s'exclame-t-il à propos du roman Le Sursis, aujourd'hui injustement déprécié. Voir Lettres de prison, ouvr. cité, p. I20, où Rebatet précise : "très remarquable réussite technique. Procédé avalé par le mouvement d'ensemble. [...] tout de même, un peu trop journalistique, reportage de grand virtuose». Hommage d'autant plus remarquable que Rebatet savait bien que Sartre n'était pas de son bord, lui qui avait prénommé... Lucien le protagoniste de «L'Enfance d'un chef", qu'il raille de son adhésion à l'extrême droite.

45. J.-P. Sartre, Saint Genet [1952], Paris, Gallimard, rééd. 1978, p. 498.

46. Ibid., p. 505 . 
Oui, ce que Sartre écrit à propos de Genet, comment ne pas l'appliquer une dernière fois à Rebatet :

Cherchant le trouble et le plaisir, Genet commence par s'envelopper dans ses images comme le putois dans son odeur. Celles-ci appellent d'elles-mêmes des mots qui les renforcent, souvent même elles demeurent inachevées, il faut des mots pour terminer l'affaire; ces mots exigent d'être prononcés et, finalement, écrits, l'écriture appelle et crée son public, le narcissiste onaniste finit par s'étancher dans les mots ${ }^{47}$.

Et les mots font lien. Genet pourra du coup s'écrier : «Ma seule victoire est verbale». Quant à Rebatet, il aura remplacé le Verbe, qui sépare, par la verve, qui se fait lire - qui réunit.

\section{Du Verbe à la verve}

Le Verbe, exposé (Régis) ou critiqué (Michel) : le roman s'alourdit. La verve : le roman s'envole. Antoine Blondin loue son «entrain prodigieux ${ }^{48}$ ». Dans ses Lettres de prison, Rebatet se présente comme « un homme à qui l'on a reconnu quelque verve» - sans doute fait-il allusion au succès des Décombres ${ }^{49}$; et il suggère une définition : "le travail d'expression fait s'épanouir la chose à exprimer ${ }^{5 \circ}{ }^{\circ}$. La verve est un épanouissement du dit dans le dire.

Les Deux Étendards essaient de maintenir ou de renouveler la verve des Décombres. Le terme de verve se présente lui-même de façon récurrente : il est avant tout appliqué à Michel, mais aussi, par trois fois, à Anne-Marie, et aux deux jeunes gens en une occurrence ${ }^{51}$. Mais jamais, sauf erreur, à Régis, l'homme du Verbe divin. Ce qui d'évidence est hautement significatif. Là encore, on trouve une définition possible : «ces débauches d'idées et de mots qui fusaient miraculeusement» (p. I86). Le miracle de la débauche, telle serait la verve...

47. Ibid., p. 500. On lirait volontiers sépancher à la place de sétancher, mais les deux font sens.

48. A. Blondin, Ma vie entre les lignes, ouvr. cité, p. I03.

49. L. Rebatet, Lettres de prison, ouvr. cité, p. 86. Quant aux modèles littéraires, la verve des Décombres fait songer à Léon Bloy (même s'il y est blâmé comme "véritable Juif d'adoption", p. IIo), et à Céline, que le pamphlet porte aux nues comme «le génie, notre seul prophète» (p. 249), tout en citant cum laude ses traits d'antisémitisme. Rebatet savait par cour des passages entiers de Bagatelles pour un massacre, et se plaisait à les réciter à haute voix. A. Yaeger Kaplan parle d'une "oralité fasciste" dans une analyse où elle articule de telles performances, les fonctions de Rebatet à Vichy en 1940 (il travaille pour le "Journal de la radio"), et l'emploi de la radio par les nazis ("Broadcasting: Rebatet», Reproductions of Banality. Fascism, Literature and French Intellectual Life, Minneapolis, University of Minnesota Press, 1986).

50. Ibid., p. 90.

5I. Michel : p. I86, 464, 584, 88I, I0I8, I049, I086; Anne-Marie : p. 447, 453, 788; les deux à la fois : p. 933 . 
Si l'on s'aide des dictionnaires de langue, on voit que le terme de verve enferme cinq traits sémantiques, qui éclairent le roman de Rebatet.

I) Littré indique un sens ancien du mot, rare : «Caprice, bizarrerie, fantaisie». Il faudrait repérer les moments de caprice dans Les Deux Étendards, ceux où le romancier semble s'élancer, derrière son personnage, sur une piste soudainement découverte : ainsi de la poursuite de Gaupette par Michel. Mais plus généralement, en tant que caprice, la verve exerce une fonction de libération. La prison : l'espace est compté. La verve : les mots se font espace, se déploient, se dé-chaînent. Une comparaison serait à mener avec la verve étonnante du roman de Jacques Perret, Le Caporal épinglé (1947) : lui aussi un - ancien - prisonnier (mais en tant que soldat français), et un homme de droite, voire d'extrême droite (royaliste).

2) Toujours selon Littré, la verve est la «chaleur d'imagination qui anime le poète, l'orateur, l'artiste dans la composition ». C'est la «fièvre incomparable» et nécessaire dont Rebatet parle à Cailleux ${ }^{52}$. Littré encore : «Il se dit quelquefois d'excitation due à d'autres impulsions que la chaleur de la composition ", par exemple le patriotisme chez Mirabeau. Ou l'anticléricalisme, chez Rebatet, et l'érotisme, qui associe la verve et la verdeur, et qui manifeste au mieux comment la verve est ce qui s'excite.

3) La chaleur d'imagination engendre deux qualités qui peuvent devenir contradictoires. D'un côté, l'abondance, la copia. Variété et richesse du lexique (des lexiques) dans Les Deux Étendards, de la théologie à l'argot en passant par l'analyse psychologique et par la critique, littéraire, picturale ou musicale ${ }^{53}$. Quel amour des mots! On a envie de dresser une liste des mots rares que le roman fait vivre ou revivre (comme maujoint, p. 704, repris à Rabelais, ou geinte, p. 703, emprunté à Verlaine, l'un des poètes préférés de Rebatet), de ceux qu'il invente aussi, comme séncatholarder (p. 595), désécration (p. 885), christianerie (p. I229), ou, tout simplement, de ceux dont il aiguise le sens sur sa meule. D'un autre côté, être en verve, c'est "manifester son esprit», et l'esprit procède par saillies, par éclairs; à la fin du XviII siècle, le mot de verve signifiait «fougue, vivacité». Mais comment soutenir cette vivacité sur mille trois cents pages?

4) L'étymologie du mot verve que Rebatet pouvait connaître n'est pas celle que propose la philologie actuelle. Littré : «Lat. verva, tête de bélier sculpté, de vervex, bélier [...]; mot pris pour caprice, fantaisie, qui est le sens ancien,

52. L. Rebatet, Lettres de prison, ouvr. cité, p. 27.

53. En ce sens, Y. Reboul me semble mieux inspiré quand il parle de «baroque célinien» que lorsqu'il évoque les «nombreux passages où s'entend en quelque sorte la voix de Gide» - laquelle, en tout état de cause, me paraît toujours plus feutrée que celle de Rebatet. 
comme caprice lui-même est pris de capra, chèvre». - Mais selon le Dictionnaire historique de la langue française, "verve" serait issu (II67) du latin populaire verva, variante de verba, "parole de fantaisie", pluriel pris pour un féminin singulier du latin classique verbum. D'où je tire deux conclusions. Les cornes du bélier, ou du bouc, sont aussi celles de Satan. Dans Les Deux Étendards, on rencontre l'expression "verve du diable» (p. I059). C'est quand il parle à Anne-Marie le langage de Lucifer que la verve de Michel se déploie (p. 873), et elle notera plus tard que la verve du garçon lui revient dès qu'il s'agit de s'en prendre au christianisme (p. I049). Pour Régis, il y a d'un côté «les bafouillages des hommes et de l'autre le Verbe de Dieu» (p. 828). Entre les deux, Michel et Rebatet inventent leur verve cornue. Et si l'on abandonne l'étymologie de Littré, la verve demeure la parole... qui n'est pas le Verbe, l'esprit qui n'est pas l'Esprit. Michel cite le in principio erat Verbum (p. 885). La verve du roman s'emploie à défaire l'autorité du Verbe, à montrer qu'il n'est que verbiage. À la fin reste la verve. C'est elle qui soutient les exercices spirituels hétérodoxes - par exemple les péchés de souvenir et d'imagination, où à l'aveu se mêle l'exaltation.

5) Verbum: dans la verve entrerait aussi ce que Leiris nommait la vocalité. Sartre a tenté de l'analyser à propos de Flaubert et du célèbre gueuloir : dans la lecture demeurerait une certaine distance entre les signes de l'auteur et le déchiffreur; mais s'il y a effet de voix, alors «le locuteur entre dans le locuté par l'oreille»; de plus, si l'on joue de sa belle voix, «il s'agit moins de prouver que de fasciner et de corrompre ${ }^{54}$ ». J'applique ces remarques à la verve des Deux Étendards : ce n'est pas l'Immaculée Conception, c'est la Conception de la Corruption... Comme Flaubert vu par Sartre, Rebatet cherche par sa verve un "équivalent écrit de la séduction oraless. Ce n'est pas le Et Verbum caro factum est du quatrième Évangile, c'est la chair qui se fait voix pour devenir verve : Et caro verva facta est. Tel serait le sens de cette verve où se mêlent profanation et profération.

On aura compris qu'à mon sens la profération pleine de verve se heurte à un obstacle : la représentation de la religion catholique, défendue ou attaquée, devient encombrante, les discussions théologiques s'étendent, s'étalent, s'attardent au détriment de l'intérêt romanesque. Michel rend visite au Révérend Chaleyssin, qui reçoit des étudiants «thalas»: «le soutanier [...] dirigeait le pilpoul» (p. 698). Trait de satire? Oui, mais dans l'ensemble du roman, ce que Rebatet déteste, le pilpoul - à savoir, dans les études talmudiques la recherche de toutes les possibilités d'application d'une loi donnée, et, par extension, une

54. J.-P. Sartre, L'Idiot de la famille, t. II [1971], Paris, Gallimard, nouv. éd. revue et complétée, I988, p. 870-87I.

55. Ibid., p. 889 . 
spéculation quintessenciée et oiseuse ${ }^{56}-$ le pilpoul donc brise, plus d'une fois, l'élan polémique même qui le vise. Revanche juive ou ironie romanesque? Je ne sais. En tout cas, le pilpoul au sujet du Verbe freine la verve, et rend d'autant plus désirables les moments érotiques. Rebatet le savait, d'où, entre autres raisons, le régime masturbatoire de son écriture? Mais la compensation opère-t-elle vraiment? Chacun en jugera, en son âme et aussi en son corps.

Pour nous, brisons là, et laissons le dernier mot à Roger Nimier : "Ce livre, on le lâche toutes les heures et on l'achève en deux jours ${ }^{57}$."

56. Y. Reboul donne ces explications dans la note in de son article.

57. Journées de lectures, ouvr. cité, p. 243. 